erhalten. Nach Andés ${ }^{\circledR}$ ) findet das Verdicken von Leinöl beim Erhitzen mit Alkalien statt infolge Bildung von Polymerisationsprodukten, verursacht durch die entstehende Seife. J. Becker ${ }^{8}$ ) erzeugt nach D. R. P. 170533 aliphatische Ketone aus fettsauren Kalksalzen durch Erhitzen der letzteren im Kohlensäurestrom, da das bei $500^{\circ}$ entstehende $\mathrm{CaO}$ zirka $10-20 \%$ des entstehenden Azetons unter Bildung von Kondensationsprodukten verbraucht. Nach dem D. R. P.170 563 gewinnt H. Winter ${ }^{3}$ ) die niederen Fettsäuren aus Kokosöl und Kernöl dadurch, dass er zunächst nur bis zu $90 \%$ verseift, das Glyzerin abscheidet, dann mit $\mathrm{H}_{2} \mathrm{SO}_{4}$ zerlegt und die Fettsäuren im Vakuum mit Dampf destilliert. Emil Lonner") bewirkt die Fettlöslichkeit der Salizylsäure, indem er sie mit wässrig-alkoholischer Lösung von Fetron (Gemenge von Stearinsäureanilid, Vaseline und Lanolin) mischt.

6. Apparate. Eine Vorrichtung zur Erhitzung von Leinöl wurde von J. Buchanan ${ }^{5}$ ) beschrieben. Zur Entfernung der schleimigen Ausscheidungen aus Leinöl benutzt $A$. Stelling ${ }^{6}$ ) nach D. R. P. 177693 Filter aus Papier oder Leinwand, welche mit Stoffen wie borsaure Magnesia, harzsaures oder fettsaures Mangan oder Zink imprägniert sind, durch welche die

\footnotetext{
v) R.: Chem, Rev, 1906 , p. 17

3 Chem.-Ztg. 1906, p. 563.

') Chem. Rev. 1906, p. 177.

4) D. R. P. 173789.

Chem.-Ztg., Rep. 1906, p. 297.

o) Chem.-Ztg., Rep. 1906, p. 439.
}

Flocken katalytisch abgeschieden werden sollen. Das Aufschmelzen erstarrter Oele bei Kesselwagentransporten wurde von $K . G . L a$ u b $^{1}$ ) besprochen. Ch. D. Monroe ${ }^{8}$ beschrieb eine Einrichtung zum Schmelzen und Reinigen von Butter und Fett, Flachs ${ }^{9}$ ) eine Destillationseinrichtung für Benzin, Arthur Möhrer und Fried. Edelmann-Heldrungen ${ }^{4}$ ) eine Ausstossvorrichtung für Kerzengiessmaschinen. Ein Auszug aus Hefters Technologie der Fette ${ }^{5}$ ) behandelte die Seifendrehpresse und Kompoundpresse. E. Rigdill ${ }^{6}$ ) beschrieb einen Schwefelkohlenstoffextraktionsapparat für Oele. Nach Angaben von $E$. Bertainehand u. R. Marcille $e^{7}$ sind als Apparate für neue Oelfabrikationsverfahren zu nennen: Zur Gewinnung von Olivenöl die kontinuierliche Presse von Colin, der Zentrifugalseparator von $\mathrm{Hignet}$ e, eine Nachbildung der Milchzentrifuge, der nach dem Prinzip der Florentiner Flasche gebaute Oelabscheider von Moncado. Endlich wurden von E. Pollats $\mathrm{ch} \mathrm{ek}^{8}$ ) die in der Margarinefabrikation zur Emulgierung zweier Flüssigkeiten von verschiedenem spez. Gewicht dienenden Homogenisiermaschinen der Lübecker Maschinenfabrik besprochen.

1) Chem.-Ztg., Rep. 1906, O.: Seifens.-Ztg. 1905, p. 871.

2) Chem-Ztg., Rep. 1906, p. 240.

'Chem. Rev. 1906, p. 85.

) Chem. Rev. 1906, p. 261

5) Seifenfabr. 1906, p. 948 .

o) Chem.-Ztg., Rep. 1906, p. 438.

7) Zeitschr, f. ang. Chem. 1906, p. 686, O.: Seifens, $Z$ tg. 1905 , p. 720

8) O.: Chem. Rev. 1906, p. 6.

\title{
Beitrag zur Beurteilung gelb gefärbter Kokosfette und Kokosfett-Präparate.
}

Von Dr. Otto Sachs.

Die verschiedenartigen Beurteilungen, welche Kokosnussfette in gelb gefärbtem Zustande fortgesetzt erfahren, veranlassen mich, zur Frage: »ob solche Präparate den Bestimmungen des Margarinegesetzes vom 15. Juni 1897 unterliegen oder nicht? « nochmals Stellung zu nehmen.

Im Handel befinden sich

\section{A. Naturprodukte:}

1. gelbe Kokosnussbutter, hart, gelb gefärbt durch minimalen Zusatz von unschädlichem Pflanzenfarbstoff.

2. gelbe Kokosnussbutter, gelb gefärbt durch Pflanzenfarbstoff, weich und geschmeidig gemacht durch mechanische Knetung.

\section{B. Kunstspeisefette}

(schmalzähnliche Zubereitungen):

gelb gefärbte Kokosnussbutter, geschmeidig, streichbar gemacht durch Zumischung von Pflanzenölen.

\section{Emulgierte Naturbutterimitationen:}

Pflanzen-Margarineprodukte, hergestellt aus raffiniertem Kokosnussfett, Wasser, Eigelb, Milch, Pflanzenöl, Farbstoff und Salz.

Die Ausnahmebestimmung des $\$ 1$, Abs. 4 des Margarinegesetzes, wonach, unverfälschte Fette bestimmter Tier- und Pflanzenarten unter den ihrem Ursprung entsprechenden Bezeichnungen in den Verkehr gebracht werden können *, findet auf die unter $B$. und $C$. genannten Kokosfettpräparate keine Anwendung. Hier liegen Zubereitungen im Sinne des Gesetzes, stoffliche Veränderungen der Fette und bei $C$. sogar mit Formgebung, vor. Die sub B. genannnten Produkte »Kunstspeisefette " repräsentieren Mischfette von schmalzähnlicherKonsistenz, 
welche durch Vermischen von gereinigtem Kokosfett und Pflanzenölen hergestellt sind, und die C.-Produkte sind Imitationen von Naturbutter, milchbutterähnliche Zubereitungen, hergestellt aus gereinigtem Kokosfett und anderen hierzu geeigneten Fetten und Oelen unter Mitverwendung von Magermilch zum Zwecke der Umwandlung in eine Emulsion. Hierher gehören auch die mit Wasser und Eiweiss emulgierten Präparate. Von einem Zweifel bezüglich der Klassifikation dieser Produkte kann nicht die Rede sein. Die B.- und C.-Präparate unterliegen unbedingt den Bestimmungen des Margarinegesetzes vom 15 . Juni 1897.

Wie verhält es sich aber mit den gefärbten Naturprodukten A., von denen das sub A 1 nur gefärbt, das sub A. 2 gefärbt und weich, geschmeidig gemacht ist durch mechanische Knetung? Nach meiner Meinung findet hier, weil es einheitliche, unverfälschte Fette einer bestimmten Pflanzenart sind, die Ausnahmebestimmung des $₫ 1$, Abs. 4 Anwendung, sofern die richtige Ursprungsdeklaration stattgefunden hat. Es interessiert uns zunächst die Frage: Ist Gelbfärbung eine Fälschung? In der Zugabe eines minimalen Zusatzes unschädlichen Pflanzenfarbstoffes erblicke ich keine Fälschung im Sinne des Margarinegesetzes, denn die durch den unschädlichen Pflanzenfarbstoff bewirkte Färbung, die bei Margarine gestattet und bei Milchbutter geduldet ist, bedingt auf keinen Fall eine stoffliche Veränderung. Das Natur-Kokosfett bleibt trotz der Färbung in allen seinen charakteristischen Eigenschaften, die es leicht als solches erkennen lassen, erhalten. Es hat weder eine Zumischung von Fetten, noch die Entnahme eines zugehörigen Bestandteils stattgefunden, mithin ist keinerlei stoffliche Veränderung eingetreten und trotz des Gelbseins sind alle Merkmale, die das Naturprodukt Kokosbutter kennzeichnen, dieselben geblieben. Das Fett ist deshalb als unverfälscht zu betrachten, für welches die Ausnahmebestimmung des $\int 1$, Abs. 4 des Margarinegesetzes Anwendung findet, sofern die jede Täuschung ausschliessende Ursprungsdeklaration gegeben ist. Das Gelbsein allein macht das Naturprodukt keinenfalls gleich zu einer butterschmalzähnlichen Zubereitung. Es hat wohl eine äussere Farbänderung, die eine Teilähnlichkeit bedingt, aber keine stoffliche Veränderung, die das Wesen der Zubereitung im Sinne des Gesetzes ausmacht, Platz gegriffen. Zur Feststellung der Gesamtähnlichkeit ist auch die Berücksichtigung der inneren Eigenschaften, Geruch, Geschmack und Plastizität, ein Erfordernis.
Was nun das gelb gefärbte, durch mechanische Knetung weich und geschmeidig gemachte Kokosfett anbetrifft, so erblicke ich auch in der mechanisch bewirkten Konsistenzveränderung keine Zubereitung. Der Zubereitungsbegriff im Sinne des Margarinegesetzes setzt die Herstellung von Mischungen, eventuell mit neuer Formgebung, voraus oder die Entnahme eines zugehörigen Bestandteils. Beides ist hier nicht der Fall und da alle die charakteristischen Merkmale des Naturfettes, die chemischen Konstanten, noch in vollem Umfange intakt sind und die Unverfälschtheit des Fettes chemisch erkennen lassen, so unterliegt ein solches gelbes, durch mechanische Knetung geschmeidig gemachtes Kokosfett nicht den Bestimmungen des Gesetzes, wenn Ursprung und Färbung deklariert ist.

Uebrigens ist noch darauf hinzuweisen, dass eine Konsistenzänderung schon durch die Temperatur erfolgen kann. Das gelbe harte Produkt kann bei einer $20^{\circ}$ übersteigenden Temperatur von selbst weich werden, wie anderenteils das durch mechanische Knetung weich gemachte durch längeres Lagern und Temperaturerniedrigung wieder fest und hart werden kann.

Weder die Gelbfärbung, noch die mechanische Konsistenzänderung, Knetung, können die Berechtigung geben, die gelben Naturprodukte, wenn richtig deklariert, als Zubereitungen anzusehen, für welche die Bestimmungen des Gesetzes vom 15. Juni 1897 massgebend sind.

Die Unsicherheit der Auslegung des Margarinegesetzes ist lediglich eine Folge der im Laufe der Jahre aufgetretenen grossen Missstände hinsichtlich der den Fabrikanten von Speisefetten obliegenden Deklarationspflicht. Hier wird wohl seitens der meisten Kokosbutterfabrikanten gefehlt.

Jedes Naturfett darf nur unter einem seinen Ursprung anzeigenden Namen in den Handel gebracht werden. Den Anforderungen des Gesetzes entsprechen nach meiner Meinung nur die Deklarationen gereinigtes Kokosfett, Kokosbutter, Kokosnussbutter, gereinigtes Fett der Kokosnüsse, Butter aus Kokosnüssen«.

Phantasienamen, für sich allein gebraucht, können auf keinen Fall den Konsumenten genügend über die wahre Beschaffenheit des feilgebotenen Artikels orientieren. Bezeichnungen wie Pflanzenfett, Pflanzenbutter, Pflanzennussfett, Pflanzennussbutter sind $\mathrm{zu}$ allgemein und weisen nicht auf die bestimmte Pflanzenart hin, woraus das Fett entstammt, und Benennungen wie Palmfett, Palmenfett, Palmbutter, Palmenbutter, 
Palmnussfett, Palmennussbutter, Fett aus Palmfrüchten, Butter aus Palmfrüchten sind als ungenügend $\mathrm{zu}$ verwerfen, weil sie nur auf die Allgemeinheit der Palmfrüchte hindeuten, aber nicht auf die spezielle Palmfrucht, aus welcher das Fett gewonnen ist.

Es ist deshalb anzustreben, dass der Deklaration in Zukunft eine grössere Aufmerksamkeit geschenkt wird und dass die Ausführungs-Bestimmungen über die Deklarationspflicht verschärft werden, um die Unsicherheit der Gesetzesauslegung hinwegzuräumen zum Besten der interessierten Margarine- und Kokos-
butter-Fabrikanten und vor allem zum Nutzen der Konsumenten selbst.

Zusammenfassend gebe ich mein Urteil über die gelb gefärbten Naturkokosfette dahin $a b$, dass die gelben, gereinigten Kokosfette, hart und weich, geschmeidig gemacht durch mechanische Knetung, noch als unverfailschte, einheitliche Naturfette aufzufassen sind, die den Gesetzesbestimmungen vom 15. Juni 1897 nicht unterliegen, sofern die Deklaration der Färbung und der Herkunft aus der Kokosnuss richtig gegeben ist.

\section{Ueber Neuerungen auf dem Gebiete der Mineralölanalyse und Mineralölindustrie im Jahre 1906.}

Von Dr. Leopold Singer.

(Schluss.)

\section{Apparate und Diverses.}

Gruszkiewicz) bespricht einen Apparat zur Wassergehaltsbestimmung in Rohölreservoiren; Hillmer ${ }^{2}$ ) schlägt zur Bestimmung des Gewichtsinhaltes von Reservoiren Aräomanometer vor, welche von Max Schubert in Chemnitz geliefert werden. Das Manometer zeigt den Druck der Flüssigkeitssäule im Reservoir 2n, aus dem bekannten Querschnitt des Reservoirs erhält man den Inhalt in Metertonnen. Die Rü tgerswerke ${ }^{3}$ ) patentieren einen Apparat zur Entwässerung von Teer- und Mineralölen mit grösserem spez. Gewicht als Wasser. Die diversen, teils patentierten, teils zum Patent angemeldeten Destillier- und Kolonnenapparate aufzuzählen, ist umso zweckloser, als nicht immer klar ist, ob diese Apparate für die Spiritus- oder für die Mineralölindustrie gedacht sind. Erwähnt sei eine neue Methode der fraktionierten Destillation von $\mathrm{Huglo}^{4}$ ), mit Hilfe eines Kessels mit Rührwerk und Exhaustor; ferner das Verfahren der Dampfkessel- und Gasometerfabrik Braunschweig nach Dr. Flachs ${ }^{5}$ ); dasselbe besteht im wesentlichen in einem Apparate zur Dephlegmation, um besonders bei der Benzinraffination Schutz vor Zersetzungen zu bieten. Erwähnt sej ferner ein Apparat zur kontinuierlichen Destillation von Gerhardt ${ }^{6}$ ).

1) Naphtha 1906, p. 24.

2) Petroleum 1906 , p. 413.

) D. R. P. 161524. Zeitschr. f, ang. Chem. 1906, p. 443.

4) Journ. du pétr. 1906, p. 234.

s) D. R. P. 169191 . Petroleum 1906, p. 412. Chem. Rev. 1906, p. 85. F. Pat. 363993 . Zeitschr. f. ang. Chem. 1906, p. 1154. O. Pat, 26 674. Chem.-Ztg. 1906, p. 1150.

6) D. R. P. 164515. Zeitschr. f. ang. Chem. 1906, p. 589. Petr. Rev, 1906, p. 174. Chem.-Ztg. 1906, p. 389 ,
Ein weiteres Patent von Hirzel ${ }^{1}$ ) befasst sich mit der kontinuierlichen fraktionierten Destillation von Mineralöl. Das Destillat läuft in dünner Schicht innerhalb der durch eine Scheidewand geteilten Blase unter geregeltem Zufluss des Füllmaterials hin und zurück, die zu destillierende Flüssigkeit wird in einzelne Ströme geteilt. Andere Apparate zur ununterbrochenen Destillation patentieren Fischer ${ }^{2}$ ), ferner Barbet ${ }^{3}$ ) und Forbes ${ }^{4}$. Ausserdem ist ein Apparat von de Clercqs ${ }^{5}$ ) zu erwähnen, welcher zum feuer- und schaumsicheren Destillieren von Teer dient. Im Apparat befindet sich ein Heizschlangensystem und durch eine entsprechende Einrichtung wird die Zirkulation einer stets nur geringen Flüssigkeitsmenge bewirkt. Ein deutsches Patent ${ }^{\circ}$ ) schützt ein Verfahren von Di a mand zur Regelung des Minderdruckes in Destillationsgefässen, wobei dieser genau nach Bedarf eingestellt werden und man den Auslauf der Flüssigkeit frei beobachten kann. Ueber einen neuen Dephlegmator berichtet Charitschkoff ${ }^{7}$ ), eine neue Vorrichtung mit selbstwirkender Zirkulation behufs gleichmässiger Vorwärmung proponiert Laszló $^{8}$ ), einen Apparat zur Abscheidung von Paraffin aus Paraffinöl haben

1) D. R. P. 172224. Chem. Rev. 1906, p. 266. Chem.-Ztg. Rep. 1906, p. 239

2) F. Pat. 359533. Chem,-Ztg. 1906, p. 161.

S) D. R. P. 163385 . Zeitschr. f. ang. Chem, 1906, p. 353 .

4) D. P. A. F. 18900. Chem.-Ztg. 1906, p. 26. p. 1778

5) D. R. P. 166723 . Zeitschr. f, ang. Chem, 1906 ,

o) D. R. P. 179046. Chem, Rev. 1906, p. 317.

7 Petr. Rev, 1906, Bd. XIV, p. 417.

8) Zeitschr, f. ang, Chem. 1906, p. 461. 